\title{
Indicadores de sostenibilidad urbana para la ciudad de Cuenca: Desechos residenciales sólidos y de construcción
}

\section{Urban sustainability indicators for Cuenca city: Residential solid and}

\section{construction waste}

1 José Jacinto Barbecho Benavides

https://orcid.org/0000-0003-1933-7757

Universidad Católica de Cuenca, Maestría en Construcciones con Mención en

Administración de la Construcción Sustentable, Azuay,

jose.barbecho.03@est.ucacue.edu.ec

2 Pedro Javier Angumba Aguilar

https://orcid.org/0000-0001-8327-1428

Universidad Católica de Cuenca, Maestría en Construcciones con Mención en

Administración de la Construcción Sustentable, Azuay

pangumba@ucacue.edu.ec

3 Juan Francisco Cazorla

https://orcid.org/0000-0002-4604-9470

Universidad Católica de Cuenca, Carrera de Arquitectura, Azuay,

juan.cazorla@ucacue.edu.ec

4 Juan Felipe Quesada Molina

https://orcid.org/0000-0002-6931-0192

Construcción Sustentable, Azuay

felipe.quesada@ucacue.edu.ec

Artículo de Investigación Científica y Tecnológica

Enviado: 06/12/2021

Revisado: 21/12/2021

Aceptado: 17/01/2022

Publicado:05/03/2022

DOI: https://doi.org/10.33262/concienciadigital.v5i1.2.2084

Cítese: $\quad$ Barbecho Benavides, J. J., Angumba Aguilar, P. J., Francisco Cazorla , J., \& Quesada Molina, J. F. (2022). Indicadores de sostenibilidad urbana para la ciudad de Cuenca: Desechos residenciales sólidos y de construcción. ConcienciaDigital, 5(1.2), 27-45. https://doi.org/10.33262/concienciadigital.v5i1.2.2084

CONCIENCIA DIGITAL, es una Revista Multidisciplinar, Trimestral, que se publicará en soporte electrónico tiene como misión contribuir a la formación de profesionales competentes con visión humanística y crítica que sean capaces de exponer sus resultados investigativos y científicos en la misma medida que se promueva mediante su intervención cambios positivos en la sociedad. https://concienciadigital.org

La revista es editada por la Editorial Ciencia Digital (Editorial de prestigio registrada en la Cámara Ecuatoriana de Libro con No de Afiliación 663) www.celibro.org.ec 


\section{Palabras claves:}

Sostenibilidad urbana;

Desechos

sólidos;

Construcción; Indicadores

\section{Keywords:}

Urban sustainability; Solid waste; Construction; Indicators.
Resumen

Introducción. En la ciudad de Cuenca, el desarrollo y los cambios urbanos no son distintos a los de otras localidades de la región. Donde la implementación de indicadores de sostenibilidad urbana, guían a las ciudades en la búsqueda de enfoques que promueven el desarrollo sostenible en sus tres ejes principales: económico, ambiental y social, generando beneficios al medio ambiente y a la calidad de vida de sus habitantes. Objetivo. El objetivo de esta investigación es determinar los indicadores de sostenibilidad urbana, de desechos residenciales sólidos y de construcción que se puedan aplicar a la ciudad de Cuenca. Metodología. Mediante una metodología que involucra la investigación, revisión y recopilación de literatura existente acerca de los marcos internacionales de indicadores de desarrollo sostenible. Determinación de un marco de referencia que ayudara a la elaboración de una matriz de indicadores básicos de residuos sólidos y de construcción, que posteriormente son homologados en base a su similitud de criterios en cuanto a los objetivos y metodologías de calificación, obteniendo de esta manera un set de indicadores de sostenibilidad urbana de desechos residenciales sólidos y de construcción que pueden ser aplicados a nivel local de la ciudad de Cuenca. Estos indicadores serán validados mediante un juicio de expertos que darán su aporte en cuanto a suficiencia, claridad, relevancia y coherencia de cada uno de los indicadores presentados. Resultados. Finalmente, con información obtenida de la Empresa Pública Municipal de Aseo de Cuenca - EMAC-EP, se realizará la aplicación de 4 indicadores para determinar si la cuidad presenta índices de sostenibilidad en cuanto a gestión de desechos sólidos y de construcción. Conclusión. Los resultados obtenidos dentro de la presente investigación serán de importancia debido a que contribuyen al desarrollo sostenible de la ciudad.

\section{Abstract}

Introduction. The development and urban changes in the city of Cuenca are like other locations of the region. A region where the implementation of urban sustainability indicators guides the different cities to the search of approaches that promote the sustainable development of its three main axis: economic, environmental, and social; generating benefits to the environment at the same time as well as the quality of life of its inhabitants. 
Objective. The objective of this investigation is to determine the urban sustainability indicators, construction and residential waste that could apply to Cuenca city. Methodology. This investigation is going to be develop by using a methodology that involves investigation, revision, and compilation of existent literature of the international framework about the sustainability indicators. In addition, the creation of a framework will help to design a matrix of basic indicators for construction and solid waste. Thus, these indicators are going to be homologated based on their similar criteria with respect of the initial objectives; evaluation and methodology to obtain a set of urban construction and solid waste sustainability indicators that can be applied at local level in Cuenca. These indicators are going to be valid throughout some experts' criteria that back them up in the adequacy, clearness, importance, and coherence sense of the presented indicators. Results. Finally, with the obtained information from Municipal Public Company of Clean de Cuenca-EMAC-EP, four indicators will be applied to determine whether the city presents sustainability indexes with respect to construction and solid waste management. Conclusion. The results of the presented research will be important because of its capacity to contribute to the sustainability development of the city.

\section{Introducción}

El constante crecimiento y desarrollo de las ciudades, ha generado que gran parte de la población mundial viva en las zonas urbanas, "la urbanización es comúnmente considerada como uno de los procesos sociales más importantes, teniendo enorme impacto en el medio ambiente a nivel local, regional y global" (Hiremath et al., 2013, p. 555). En la actualidad más del 56\% de la población mundial vive en asentamientos urbanos, según United Nations, Department of Economic and Social Affairs (2018), "Para 2030 se proyecta que las áreas urbanas albergaran el 60\% de personas en todo el mundo"(p. 2).

En las ciudades se genera gran cantidad de desechos residenciales sólidos y de construcción, cuya recolección y disposición final se han convertido en uno de los principales problemas ambientales de la población. "Su gravedad presenta distintos matices de acuerdo al nivel de desarrollo de los países, el tamaño de los asentamientos, la conciencia ambiental de los ciudadanos, etc." (Guerrero y Erbiti, 2004, p. 71). En el 
Ecuador y el mundo la contaminación ambiental debido a la mala gestión de los desechos sólidos, así como, de los desechos resultantes de las actividades de construcción, renovación y demolición se ha convertido en un desafío importante para los científicos, debido a que actúan como un factor desencadenante de contaminación de agua, aire y suelos. "El vertido a cielo abierto y la quema a cielo abierto son los principales sistemas de tratamiento y disposición final de residuos, principalmente visibles en los países de bajos ingresos" (Ferronato \& Torretta, 2019, p.8).

En la ciudad de Cuenca, el desarrollo y los cambios urbanos no son distintos a los de otras localidades de la región, en donde el consumo de materia prima y recursos energéticos cada vez es mayor, lo cual genera una serie de impactos ambientales. Es importante entonces comprender los conceptos de sostenibilidad urbana, según Hiremath et al. (2013):

El desarrollo urbano sostenible significa específicamente lograr un equilibrio entre el desarrollo de las áreas urbanas y la protección del medio ambiente con miras a la equidad en los ingresos, el empleo, la vivienda, servicios básicos, infraestructura social y transporte en las áreas urbanas. (p. 556)

Por esta razón, a nivel internacional, se ha propuesto la implementación de indicadores para la medición y monitoreo (Hák et al., 2016; Sachs, 2012), de temáticas que guían y evalúan el progreso de las ciudades hacia el "Desarrollo Sostenible" (Hassan y Lee, 2015, p. 199).

Los indicadores son medidas cuantitativas, cualitativas o descriptivas que informan sobre la situación de entornos urbanos y la incidencia de sus procesos sobre el medio ambiente, también ayudan en la planificación de ciudades que ofrezca oportunidades para todos, con acceso a servicios básicos de energía, vivienda, transporte, espacios públicos y que, al mismo tiempo controlen el uso de recursos y reduzcan los impactos ambientales. Sin embargo, la literatura científica no ha podido definir como estos indicadores pueden ser trasladados a diferentes contextos, disminuyendo la escala de estudio, es por esto por lo que, a nivel local de la ciudad de Cuenca, aún no existe un marco sólido de indicadores que guie a una correcta gestión de desechos residenciales sólidos y de construcción, encaminando a la cuidad al desarrollo sostenible. ¿Se puede desarrollar un marco de indicadores de sostenibilidad urbana en relación con la gestión de desechos residenciales sólidos y de construcción aplicados a la ciudad de Cuenca? La presente investigación responde a la necesidad de superar la brecha del conocimiento mediante el desarrollo de marcos de indicadores de sostenibilidad urbana, que pueden ser aplicados a nivel local.

Con el objetivo de obtener indicadores de gestión de desechos residenciales sólidos y de construcción, que permita su uso, aplicación y sirva dentro de la planificación urbana, para la generación de estrategias y políticas que encaminen a la ciudad al desarrollo 
sostenible. El trabajo que aquí se presenta se desarrollara con la aplicación de una metodología que involucre la investigación, revisión y recopilación de literatura existente acerca de los marcos internacionales de indicadores de desarrollo sostenible, para luego preceder con la selección de indicadores de desempeño apropiados para la ciudad de Cuenca, en relación a la gestión de desechos residenciales sólidos y de construcción, determinando de esta manera marcos solidos de indicadores locales, que pertenezcan a marcos internacionales para que sean ampliamente aceptados para la evaluación de la sostenibilidad. Los resultados obtenidos serán de gran importancia para el desarrollo de investigaciones complementarias dentro del campo de desarrollo sostenible de las ciudades, así como también contribuirán a la generación de impactos ambientales positivos, al aportar con información que permita un correcto manejo de desechos sólidos y de construcción.

"Los principales desafíos urbanos están relacionados con el crecimiento urbano no planificado y los servicios públicos de mala calidad, que generan una falta de compromiso para hacer cumplir las leyes y lograr los objetivos de desarrollo sostenible" (Moschen et al., 2019, p.891). "Potenciar las ventajas de cada territorio constituye la idea central del desarrollo endógeno. Para su concreción práctica resultará imprescindible la instrumentalización de un adecuado uso y gestión del suelo" (Quezada Ortega et al., 2021, p. 115). Es ahí donde los indicadores juegan un papel de suma importancia

\section{CRITERIOS DE SOSTENIBILIDAD URBANA}

\section{Ciudades Sostenibles}

Una ciudad sostenible es una ciudad que reduce el impacto ambiental de sus actividades y promueve modalidades de consumo y producción sostenibles, acordes con sus propias condiciones territoriales, geográficas, sociales, económicas y culturales. Según Ahvenniemi et al. (2017), "las ciudades tienen un papel clave en la lucha contra el cambio climático" (p. 234). Es así que durante los últimos años el diseño y el desarrollo urbano se ha considerado como el instrumento para dar forma al futuro de la ciudad, de ahí que según Ameen et al. (2015), "la evaluación de la sostenibilidad del diseño y desarrollo urbano se considera cada vez más indispensable para la toma de decisiones informada" (p. 110). Mismas que guían a las ciudades a desarrollar estrategias y objetivos encaminados hacia el desarrollo sostenible.

\section{Sostenibilidad urbana}

Durante la última década el concepto de desarrollo sostenible ha tomado fuerza, la sostenibilidad urbana debe ser entendida como la búsqueda de un desarrollo que no degrade el entorno y a su vez proporcione calidad de vida a los ciudadanos. La evaluación de la sostenibilidad ayuda a determinar cómo las ciudades se vuelven más sostenibles, 
para esta evaluación a lo largo del tiempo han surgido diferentes métodos, técnicas e instrumentos entre uno de ellos los basados en indicadores que integran el desarrollo y la protección del medio ambiente.

\section{Indicadores de sostenibilidad urbana}

Los indicadores de sostenibilidad monitorean el progreso hacia los Objetivos de Desarrollo Sostenible (ODS). En la mayoría de las herramientas de evaluación, se pueden agrupar en dos: indicadores urbanos comunes y locales, que reflejan las prioridades locales e internacionales para la sostenibilidad urbana. "Sin embargo, no existe una lista específica de indicadores que se adapte a todos los países, regiones y comunidades" (Ameen et al., 2015, p.113). Por lo tanto, los indicadores para medir la sostenibilidad deberían ser adecuados para las condiciones específicas de cada localidad, y al mismo tiempo, estar de acuerdo con los Objetivos universales de Desarrollo Sostenible. Según Dizdaroglu (2015), la evaluación de sostenibilidad basada en indicadores "proporciona información sobre el estado del medio ambiente al producir un valor cuantitativo" (p. 119). Los datos encontrados son necesarios para guiar hacia mejores políticas que contribuyan a la sostenibilidad urbana.

\section{Metodología}

La metodología por desarrollar dentro de la presente investigación se constituye como una herramienta fundamental ya que permitirá aportar al conocimiento y alcanzar los objetivos planteados. Para lo cual se analizaron las características generales de metodologías utilizadas y sistemas de calificación de métodos de evaluación, para poder obtener resultados precisos dentro de la investigación. De ahí que el estudio se enmarca en un enfoque metodológico de tipo no experimental, descriptivo y analítico y sigue la propuesta metodológica de (Velásquez, 2013, p. 20). Que se desarrolla en 5 etapas; las cuales se presentan a continuación:

\section{1.- Determinar las limitaciones del estudio mediante un marco de referencia.}

El campo de estudio se limita a 6 documentos de marcos teóricos de mayor influencia a nivel internacional (Tabla 1), los cuales en su contenido definen indicadores para la evaluación de la sostenibilidad de las ciudades que son ampliamente reconocidos a nivel internacional. 


\section{Tabla 1}

\section{Marcos teóricos considerados en el estudio}

\begin{tabular}{|c|c|c|}
\hline & Marco Teórico & Descripción \\
\hline 1 & $\begin{array}{l}\text { Objetivo de desarrollo } \\
\text { sostenible } 11 .\end{array}$ & $\begin{array}{l}\text { Los ODS abordan, de manera integrada, las dimensiones sociales, } \\
\text { económicas y ambientales del desarrollo, los datos y las métricas } \\
\text { permiten a las ciudades tomar decisiones correctas que apoyen a } \\
\text { la consecución de las metas del ODS } 11 \text {. }\end{array}$ \\
\hline 2 & $\begin{array}{l}\text { ISO } 37120 \text { _ Indicadores de } \\
\text { servicios de la ciudad y } \\
\quad \text { calidad de vida. }\end{array}$ & $\begin{array}{l}\text { Un estándar con un conjunto de indicadores que evalúan el } \\
\text { desempeño de la prestación de servicios de las ciudades y la } \\
\text { calidad de vida con el fin de proporcionar un enfoque holístico e } \\
\text { integrado para el desarrollo sostenible y la resiliencia. }\end{array}$ \\
\hline 3 & $\begin{array}{l}\text { Guía Metodológica_Iniciativa } \\
\text { ciudades emergentes y } \\
\text { sostenibles. }\end{array}$ & $\begin{array}{l}\text { Una metodología de rápida aplicación y diagnóstico que facilita a } \\
\text { las ciudades la formulación de planes de acción mediante la } \\
\text { identificación de intervenciones estratégicas que contribuyan al } \\
\text { logro de sus metas de sostenibilidad en el corto, mediano y largo } \\
\text { plazo. }\end{array}$ \\
\hline 4 & $\begin{array}{l}\text { LEED v4.1 Ciudades y } \\
\text { Comunidades: Planificar y } \\
\text { Diseñar. }\end{array}$ & $\begin{array}{l}\text { Un concepto de certificación verde aplicado al contexto de la } \\
\text { ciudad o comunidad, que contiene un conjunto de estándares } \\
\text { medibles que identifican si el desarrollo es ambientalmente } \\
\text { superior. }\end{array}$ \\
\hline 5 & BREEAM COMUNITIES & $\begin{array}{l}\text { Un método de evaluación que proporciona "una forma de mejorar, } \\
\text { medir y certificar la sostenibilidad social, ambiental y económica } \\
\text { de los planes de desarrollo a gran escala". }\end{array}$ \\
\hline 6 & $\begin{array}{c}\text { CASBEE CITY_ } \\
\text { Manual_2012 }\end{array}$ & $\begin{array}{l}\text { Una herramienta de evaluación del desempeño ambiental a escala } \\
\text { urbana centrada en los fenómenos que podrían ser consecuencia } \\
\text { de la conglomeración de edificios. }\end{array}$ \\
\hline
\end{tabular}

Fuente: Elaboración propia a partir de marcos teóricos de estudio.

\section{2.- Investigación bibliográfica}

La revisión de fuentes secundarias y terciarias de datos y literatura existente disponible en fuentes científicas de bases digitales, ha permitido identificar formalmente el estado del arte, así también de la revisión de los marcos teóricos utilizados en el estudio se pueden obtener una primera selección de indicadores "Matriz de indicadores básicos" (Velásquez, 2013, p. 24), mismos que están ordenados de manera descendente de acuerdo al nivel de importancia que presta cada una de las herramientas a la gestión de desechos residenciales sólidos y de construcción (Tabla 2), indicadores que serán de utilidad para las etapas posteriores en el desarrollo del presente trabajo de investigación. 


\section{Tabla 2}

Matriz de indicadores básicos

\begin{tabular}{clc}
\hline $\begin{array}{c}\text { Nivel de } \\
\text { Importancia }\end{array}$ & \multicolumn{1}{c}{ Nombre del Marco Teórico - Herramienta } & $\begin{array}{c}\text { Numero de } \\
\text { Indicadores }\end{array}$ \\
\hline 1 & Guía Metodológica_Iniciativa ciudades emergentes y sostenibles. & 7 \\
2 & ISO 37120__Indicadores de servicios de la ciudad y calidad de vida. & 6 \\
3 & LEED v4.1 Ciudades y Comunidades: Planificar y Diseñar. & 5 \\
4 & BREEAM COMUNITIES & 4 \\
5 & Objetivo de desarrollo sostenible 11. & 2 \\
6 & CASBEE CITY__Manual_2012 & 1 \\
\hline
\end{tabular}

Fuente: Elaboración propia a partir de marcos teóricos de estudio.

3.- Homologación "Selección y elaboración de un set de indicadores de sostenibilidad de gestión de desechos sólidos y de construcción”.

Con la muestra de indicadores de sostenibilidad, previamente definidos y en base a un análisis crítico de los aspectos relevantes de igualdad o equivalencia entre indicadores de las distintas herramientas se proponen 10 indicadores de gestión de desechos residenciales sólidos y de construcción que pueden ser aplicados a nivel de ciudad (Tabla 3), que a su vez se agrupan dentro de las tres dimensiones de la sostenibilidad: ambiental, económica y social.

\section{Tabla 3}

Set de indicadores de sostenibilidad de gestión de desechos sólidos y de construcción

\begin{tabular}{clll}
\hline No & \multicolumn{1}{c}{ Indicador } & \multicolumn{1}{c}{ Objetivo } & \multicolumn{1}{c}{ Método de evaluación } \\
\hline 1 & $\begin{array}{l}\text { Porcentaje de } \\
\text { residuos sólidos } \\
\text { urbanos, con } \\
\text { recolección regular } \\
\text { y descarga final } \\
\text { adecuada. }\end{array}$ & $\begin{array}{l}\text { Determinar el porcentaje de } \\
\text { residuos sólidos urbanos } \\
\text { recolectados y con descarga } \\
\text { final adecuada al menos una vez } \\
\text { por semana. }\end{array}$ & $\begin{array}{l}\text { Porcentaje de la población de la } \\
\text { ciudad con recolección de residuos } \\
\text { sólidos al menos una vez por semana. }\end{array}$ \\
$\begin{array}{l}\text { Utilización de } \\
\text { materiales locales } \\
\text { de bajo impacto, en } \\
\text { la construcción. }\end{array}$ & $\begin{array}{l}\text { Reducir el impacto ambiental, } \\
\text { mediante el uso de materiales de } \\
\text { bajo impacto en la construcción. }\end{array}$ & $\begin{array}{l}\text { Porcentaje de materiales de bajo } \\
\text { impacto utilizados en la construcción }\end{array}$ \\
\hline
\end{tabular}




\section{Tabla 3}

Set de indicadores de sostenibilidad de gestión de desechos sólidos y de construcción (continuación)

\begin{tabular}{|c|c|c|c|}
\hline No & Indicador & Objetivo & Método de evaluación \\
\hline 3 & $\begin{array}{l}\text { Porcentaje de } \\
\text { residuos sólidos } \\
\text { urbanos, que son } \\
\text { separados y } \\
\text { clasificados para } \\
\text { reciclado. }\end{array}$ & $\begin{array}{l}\text { Maximizar el ciclo de vida de } \\
\text { los rellenos sanitarios, mediante } \\
\text { prácticas de reciclaje de } \\
\text { residuos, para transformarlos en } \\
\text { nuevos productos de } \\
\text { conformidad con los permisos y } \\
\text { normas de la ciudad. }\end{array}$ & $\begin{array}{l}\text { Se calculará como la cantidad total de } \\
\text { residuos sólidos de la ciudad que se } \\
\text { recicla en toneladas (numerador) } \\
\text { dividida por la cantidad total de } \\
\text { residuos sólidos producidos en la } \\
\text { ciudad en toneladas (denominador). A } \\
\text { continuación, el resultado se } \\
\text { multiplicará por } 100 \text { y se expresará } \\
\text { como porcentaje. }\end{array}$ \\
\hline 4 & $\begin{array}{l}\text { Porcentaje de } \\
\text { residuos sólidos de } \\
\text { la ciudad que se } \\
\text { eliminan en un } \\
\text { relleno sanitario }\end{array}$ & $\begin{array}{l}\text { Determinar el porcentaje de } \\
\text { residuos sólidos urbanos de la } \\
\text { ciudad vertidos en rellenos } \\
\text { sanitarios. El relleno debe } \\
\text { contar con sistemas de } \\
\text { tratamiento y recolección de } \\
\text { lixiviados y gas residual para ser } \\
\text { considerado sanitario }\end{array}$ & $\begin{array}{l}\text { Se calculará como la cantidad de } \\
\text { desechos sólidos de la ciudad que se } \\
\text { eliminan en un relleno sanitario en } \\
\text { toneladas (numerador) dividido por la } \\
\text { cantidad total de sólidos residuos } \\
\text { producidos en la ciudad en toneladas } \\
\text { (denominador). El resultado será } \\
\text { entonces multiplicado por } 100 \text { y } \\
\text { expresado como porcentaje. }\end{array}$ \\
\hline 5 & $\begin{array}{l}\text { Porcentaje de } \\
\text { residuos sólidos de } \\
\text { la ciudad que son } \\
\text { eliminados en } \\
\text { vertederos a cielo } \\
\text { abierto y quemados. }\end{array}$ & $\begin{array}{l}\text { Determinar el porcentaje de } \\
\text { residuos sólidos urbanos que son } \\
\text { quemados al aire libre o } \\
\text { eliminado en vertederos a cielo } \\
\text { abierto. }\end{array}$ & $\begin{array}{l}\text { Se calculará como la cantidad de } \\
\text { desechos sólidos de la ciudad que se } \\
\text { queman o son eliminados a cielo } \\
\text { abierto en toneladas (numerador) } \\
\text { dividida por la cantidad total de } \\
\text { desechos sólidos producidos en la } \\
\text { ciudad en toneladas. (Denominador). } \\
\text { A continuación, el resultado se } \\
\text { multiplicará por } 100 \text { y se expresará } \\
\text { como porcentaje. }\end{array}$ \\
\hline 6 & $\begin{array}{l}\text { Porcentaje de } \\
\text { residuos sólidos } \\
\text { urbanos que son } \\
\text { comportados }\end{array}$ & $\begin{array}{l}\text { Determinar el porcentaje de } \\
\text { residuos sólidos urbanos que son } \\
\text { tratados por compostaje }\end{array}$ & $\begin{array}{l}\text { Deberá calcularse como la cantidad } \\
\text { total de desechos sólidos de la ciudad } \\
\text { que se eliminan por otros medios en } \\
\text { toneladas (numerador) dividido por la } \\
\text { cantidad total de desechos sólidos } \\
\text { producidos en la ciudad en toneladas } \\
\text { (denominador). El resultado luego se } \\
\text { multiplicará por } 100 \text { y expresado } \\
\text { como porcentaje. }\end{array}$ \\
\hline
\end{tabular}




\section{Tabla 3}

Set de indicadores de sostenibilidad de gestión de desechos sólidos y de construcción (continuación)

\begin{tabular}{|c|c|c|c|}
\hline No & Indicador & Objetivo & Método de evaluación \\
\hline 7 & $\begin{array}{l}\text { Porcentaje de } \\
\text { residuos sólidos de } \\
\text { la ciudad que son } \\
\text { utilizados como } \\
\text { recurso energético }\end{array}$ & $\begin{array}{l}\text { Determinar el porcentaje de los } \\
\text { residuos sólidos de la ciudad } \\
\text { donde se recupera y utiliza el } \\
\text { gas del relleno sanitario para } \\
\text { generar energía o calor. }\end{array}$ & $\begin{array}{l}\text { Se calculará como la cantidad de } \\
\text { desechos sólidos de la ciudad que se } \\
\text { utiliza como recurso energético en } \\
\text { toneladas (numerador) dividida por la } \\
\text { cantidad total de desechos sólidos } \\
\text { producidos en la ciudad en toneladas. } \\
\text { (Denominador). A continuación, el } \\
\text { resultado se multiplicará por } 100 \text { y se } \\
\text { expresará como porcentaje. }\end{array}$ \\
\hline 8 & $\begin{array}{l}\text { Manejo de residuos } \\
\text { sólidos en la } \\
\text { actividad de la } \\
\text { construcción }\end{array}$ & $\begin{array}{l}\text { Reducir los daños } \\
\text { medioambientales asociados con } \\
\text { la contaminación procedente de } \\
\text { las actividades de construcción. }\end{array}$ & $\begin{array}{l}\text { Crear e implementar un plan el } \\
\text { control de erosión y sedimentación } \\
\text { para todas las actividades de } \\
\text { construcción. El plan deberá describir } \\
\text { las medidas que deben aplicarse. }\end{array}$ \\
\hline
\end{tabular}

9 Manejo eficiente de Promover la eficiencia de los residuos de construcción y demolición.

10 Programas de gestión de residuos. recursos mediante la reducción

de residuos durante la

construcción y posterior

eliminación en los vertederos

Mejorar la eficiencia del

programa de gestión de residuos

con la adopción de políticas de

apoyo que permitan desarrollar

programas inteligentes de

gestión de residuos de la ciudad.
Desarrollar un plan de gestión de residuos de construcción y demolición que contenga la narrativa de los detalles de instalaciones donde se procesan los $\mathrm{C}$ y D.

Criterio de evaluación según código de viviendas sostenibles. 3 puntos $=$ 2 estrellas. 4_5 Puntos $=4$ estrellas. 6 puntos $=5$ estrellas.

Fuente: Elaboración propia en base a datos de fuentes primarias de información

\section{4.- Validación de indicadores seleccionados por juicio de expertos.}

El juicio de expertos es un método de validación que se utiliza para verificar la fiabilidad de una investigación, que se define como: "Una opinión informada de personas con trayectoria en el tema, que son reconocidas por otros como expertos cualificados en éste, y que pueden dar información, evidencia, juicios y valoraciones" (Escobar-Pérez y Cuervo-Martínez, 2008, p. 29). Por lo expuesto el set de indicadores obtenido anteriormente se somete al juicio de expertos para su respectiva validación, el proceso 
que sigue será el propuesto en el documento "Validez de contenido y juicio de expertos: Una aproximación a su utilización”(Escobar-Pérez y Cuervo-Martínez, 2008, p. 27).

\section{1.- Definir el objetivo del juicio de expertos.}

Validar los indicadores obtenidos previamente, resultantes de la revisión de los marcos teóricos internacionales y que pueden ser aplicados a la ciudad de Cuenca para evaluar la sostenibilidad en cuanto a desechos residenciales sólidos y de construcción.

\section{2.- Selección de Jueces.}

Para la validación de indicadores en el presente estudio los jueces serán seleccionados en base al cumplimiento de las siguientes características, así como de la realización previa de una encuesta digital para establecer el conocimiento en el tema:

- Poseer mínimo título de tercer nivel (Arquitecto, Ing. Civil, Ing. Ambiental, Ing. Eléctrico, Sociólogo)

- Experiencia 5 años en la realización Investigaciones, Publicaciones o academia

- Haber trabajo procesos de gestión de desechos en la parte pública y privada.

- Experiencia en temas relacionados a gestión de desechos sólidos y de construcción.

Para el juicio de expertos se contó con la colaboración de 5 jueces, mismos que a más de cumplir con las características antes mencionadas, cuentan con disponibilidad, motivación para participar e imparcialidad, criterios que también dan realce a la selección para formar el grupo de expertos. Los participantes seleccionados completaron de evaluación que se les fue enviada.

\section{3.- Explicar tanto las dimensiones como los indicadores que están midiendo cada uno de los ítems de la prueba.}

Los indicadores que se presentan para la evolución miden la sostenibilidad en cuanto a la gestión de desechos residenciales sólidos y de construcción a nivel local de la ciudad de Cuenca.

\section{4.- Especificar el objetivo de la prueba.}

Los resultados obtenidos en el juicio de expertos permitirán desarrollar un marco de indicadores locales de sostenibilidad urbana adecuados que puedan ser aplicados a nivel local de la cuidad en cuanto a desechos residenciales sólidos y de construcción. 


\section{5.- Diseño de Plantillas.}

Se toma el diseño según: Escobar-Pérez y Cuervo-Martínez (2008), "Plantilla Juicio de Expertos" (p. 35). Misma que en su contenido califica la suficiencia, claridad, coherencia, relevancia del contenido de los objetivos, metodologías de evaluación y niveles de exigencia de cada uno de los indicadores propuestos (Tabla 4).

\section{Tabla 4}

Criterios para evaluación mediante juicio de expertos

\section{Criterios por evaluar}

\begin{tabular}{ll}
\hline Suficiencia & $\begin{array}{l}\text { Los ítems que pertenecen a una misma dimensión bastan para obtener la medición } \\
\text { de ésta. }\end{array}$ \\
Claridad & El ítem se comprende fácilmente, es decir, su sintáctica y semántica son adecuadas. \\
Coherencia & El ítem tiene relación lógica con la dimensión o indicador que está midiendo. \\
Relevancia & El ítem es esencial o importante, es decir debe ser incluido.
\end{tabular}

1. No cumple con el criterio; 2. Bajo nivel; 3. Moderado nivel; 4. Alto nivel

\section{Fuente: Escobar-Pérez y Cuervo-Martínez (2008)}

\section{6.- Calcular la concordancia entre jueces.}

En el análisis estadístico de los resultados se empleó el coeficiente de concordancia W de Kendall que indica el grado de asociación o nivel de concordancia, donde se toman valores en un "rango de $0-1$, en el que el valor 1 representa un nivel de concordancia total y 0 un desacuerdo total" (Dorantes et al., 2016, p. 332). Los datos obtenidos mediante el análisis indican que el nivel es significativo entre los rangos, pues se puede observar que en algunos casos excede el valor crítico de 0.05 , por lo cual se estima una concordancia entre las respuestas de los jueces lo que a su vez muestra que los indicadores obtenidos son adecuados, aunque susceptibles de mejora. (Tabla 5)

\section{Tabla 5}

Cálculo de concordancia - W de Kendall

\begin{tabular}{cccccc}
\hline & \multicolumn{5}{c}{ Concordancia } \\
\hline Indicador & W de Kendall & $\mathrm{p}$ & Indicador & W de Kendall & $\mathrm{p}$ \\
1 & 0.87 & $\mathrm{P}<0.05$ & 6 & 0.05 & $\mathrm{P}<0.05$ \\
2 & 0.78 & $\mathrm{P}<0.05$ & 7 & 0.76 & $\mathrm{P}<0.05$ \\
\hline
\end{tabular}




\section{Tabla 5}

Cálculo de concordancia - W de Kendall (continuación)

\begin{tabular}{cccccc}
\hline \multicolumn{5}{c}{ Concordancia } \\
\hline Indicador & W de Kendall & $\mathrm{p}$ & Indicador & W de Kendall & $\mathrm{p}$ \\
3 & 0.14 & $\mathrm{P}<0.05$ & 8 & 0.86 & $\mathrm{P}<0.05$ \\
4 & 0.07 & $\mathrm{P}<0.05$ & 9 & 0.75 & $\mathrm{P}<0.05$ \\
5 & 1.00 & $\mathrm{P}<0.05$ & 10 & 0.01 & $\mathrm{P}<0.05$ \\
\hline
\end{tabular}

Fuente: Dorantes et al. (2016)

En general se percibe una amplia gama de valores intermedios de concordancia entre los jueces, misma que da una media de 0.529 , donde indicador 5 , que hace referencia a porcentaje de residuos sólidos de la ciudad que son eliminados en vertederos a cielo abierto y quemados, es el único que cuenta con un nivel de concordancia absoluta con un valor de 1, en tanto que el nivel más bajo lo obtuvo el indicador 10 que hace referencia a programas de gestión de residuos, con un valor de 0.01 .

\section{5.- Análisis de resultados obtenidos.}

Una vez validos los indicadores por el juicio de expertos se procede con la evaluación de cuatro de ellos, para esto se toman los datos de acceso a la información pública obtenidos de la página digital oficial de la Empresa Pública Municipal de Aseo de Cuenca - EMAC - EP, en su aparatado de Rendición de cuentas 2020, ¡Las cosas claras i

Donde menciona:

La cobertura de recolección en el Cantón Cuenca es de $92.60 \%$ (Base Calculo de la EMAC-EP a 2019)

La zona que le denominamos consolida circunscribe el área urbana que incluye a centros urbanos parroquiales y áreas rurales en el entorno de la ciudad de Cuenca, y es atendida por la EMAC EP en su totalidad, por lo que se garantiza la prestación del servicio en toda extensión, logrando una cobertura del $100 \%$ en este sector (Tabla 6).

La EMAC EP tiene dos frecuencias de recolección en toda el área consolidada de la ciudad (zona urbana y varias cabeceras parroquiales): lunes, miércoles y viernes; martes, jueves y sábados. Los miércoles y jueves se realiza la recolección diferenciada. 


\section{Tabla 8}

Análisis de indicador de porcentaje de residuos sólidos urbanos que son compostados

\begin{tabular}{|c|c|c|c|c|c|}
\hline Indicador & Evaluación & Unidad & Nivele & de Ex & encia \\
\hline \multirow{2}{*}{$\begin{array}{l}\text { Porcentaje de residuos sólidos } \\
\text { urbanos que son compostados. }\end{array}$} & $=405 \times 100=0.2611$ & \multirow[t]{2}{*}{$\%$} & \multirow[t]{2}{*}{$>20 \%$} & \multirow{2}{*}{\multicolumn{2}{|c|}{$\begin{array}{l}5 \%- \\
20 \%\end{array}$}} \\
\hline & $155.104,05$ & & & & \\
\hline
\end{tabular}

Resultado $=0.26 \%$

Nota: Puntaje de nivel de exigencia, acorde al documento: Guía Metodológica. Iniciativa ciudades emergentes y sostenibles

La Empresa Pública Municipal de Aseo de Cuenca - EMAC - EP, tiene bajo su responsabilidad la Gestión del Complejo Ambiental y Humano Pichacay y entre sus componentes se encuentra el Relleno Sanitario. Para la operación del Relleno Sanitario, se cuenta con la respectiva licencia ambiental, misma que fue otorgada por el Ministerio del Ambiente, el 10 de diciembre de 2002, mediante resolución No. 078. El promedio diario de desechos que se disponen en el relleno sanitario es de entre 433 - 497,07 toneladas (Tabla 9).

\section{Tabla 9}

Análisis de indicador de porcentaje de residuos sólidos de la ciudad que se eliminan en un relleno sanitario

\begin{tabular}{|c|c|c|c|c|c|}
\hline Indicador & Evaluación & Unidad & Nive & de Exi & ncia \\
\hline $\begin{array}{l}\text { Porcentaje de residuos sólidos de la ciudad que } \\
\text { se eliminan en un relleno sanitario. }\end{array}$ & $\begin{array}{c}\text { Resultado } \\
100 \%\end{array}$ & $\%$ & $\begin{array}{l}90 \%- \\
100 \%\end{array}$ & $\begin{array}{l}80 \%- \\
90 \%\end{array}$ & $\begin{array}{c}< \\
80 \%\end{array}$ \\
\hline
\end{tabular}

Nota: Puntaje de nivel de exigencia, acorde al documento: Guía Metodológica. Iniciativa ciudades emergentes y sostenibles

\section{Resultados}

De la tabla 2 presentada en el apartado correspondiente a metodología se puede definir que la herramienta CASBEE CITY_ Manual_2012, no presenta en su contenido un análisis dirigido hacia la gestión de residuos sólidos urbanos, así como de construcción.

Una vez realizada la homologación de indicadores que se pueden aplicar a la ciudad de Cuenca en relación a la gestión de residuos residenciales sólidos urbanos y de construcción que se muestran anteriormente en la tabla 3, dichos indicadores se pueden clasificar dentro de "los tres pilares de la sostenibilidad" (Quesada, 2018, p. 53). Lo cual 
se refleja en un gráfico (Figura1), expresado en porcentajes, para visualizar la importancia de que dan las herramientas a la dimensión ambiental debido a que el tema que se desarrolla en la presente investigación se relaciona directamente a esta dimensión de la sostenibilidad.

\section{Figura 1}

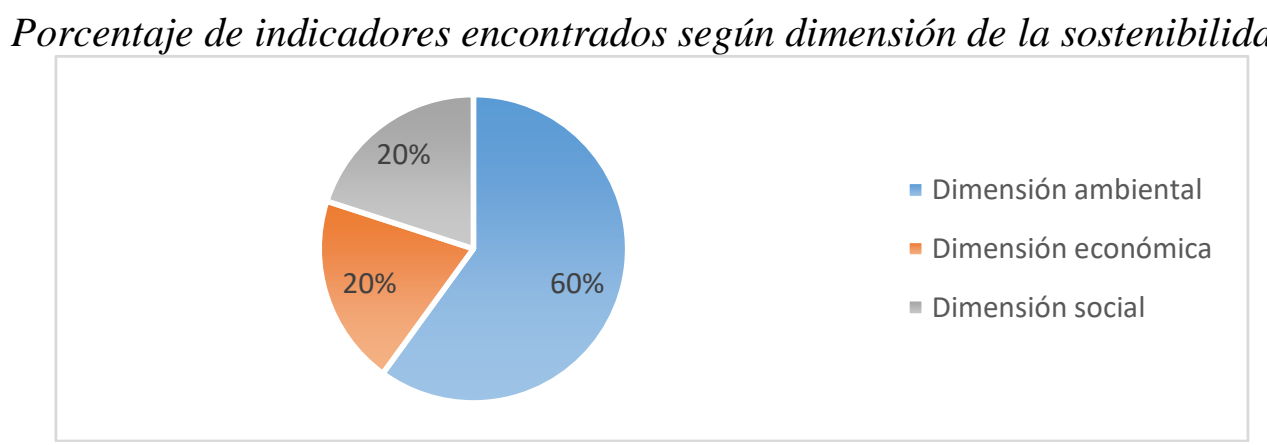

Fuente: Quesada (2018)

Del grupo de indicadores analizados en conjunto con datos de la Empresa Pública Municipal de Aseo de Cuenca - EMAC - EP, se puede observar que, si cumplen con los rangos establecidos dentro del nivel de exigencia para cada uno de los indicadores, por lo que la cuidad de Cuenca si presenta índices de sostenibilidad en cuanto a manejo y gestión de desechos residenciales sólidos y de construcción.

\section{Discusión}

Es importante reconocer que aún existe una brecha importante en cuanto a conocimiento acerca de indicadores de desarrollo sostenible en diferentes temáticas, que puedan ser aplicados a diferentes localidades, debido a que es un proceso para el cual no existe un consenso sobre una metodología definida. Sin embargo, en la presente investigación se desarrolló una propuesta metodológica que puede servir de guía para desarrollar indicadores de sostenibilidad en diferentes temáticas ya que ha aportado de manera satisfactoria para conseguir el objetivo principal del presente trabajo. Cabe resaltar que la propuesta metodología resultante dentro de la investigación puede ser aplicada a diferentes localidades o ciudades con diferentes objetivos de desarrollo sostenible.

\section{Conclusiones}

- Con los resultados obtenidos, se ha podido determinar que la ciudad de Cuenca si presenta índices adecuados de sostenibilidad en relación con desechos residenciales sólidos y de construcción, lo que a su vez responde a la interrogante planteada inicialmente. "¿Se puede desarrollar un marco de indicadores de sostenibilidad urbana en relación con la gestión de desechos residenciales sólidos y de construcción aplicados a la ciudad de Cuenca?" 
- Los indicadores resultantes contribuyen a la evaluación de la sostenibilidad de la ciudad de Cuenca en cuanto a desechos residenciales sólidos y de construcción, mismas que ayudaran a la planificación y toma de decisiones dentro de la Ilustre Municipalidad de Cuenca. Los resultados obtenidos con la aplicación de indicadores deben ser discutidos tanto al interior como al exterior de los municipios, ya que la información recatada será de gran utilidad para otras instituciones a nivel regional o nacional.

\section{Agradecimientos}

El presente artículo es parte del trabajo de investigación y titulación del Programa de Maestría en Construcción con Mención en Administración de la Construcción Sustentable de la Universidad Católica de Cuenca, vinculados al Proyecto de Investigación: INDICADORES DE SOSTENIBILIDAD URBANA PARA LA CIUDAD DE CUENCA - ECUADOR, por ello agradecemos a todos y cada uno de los instructores pertenecientes a los grupos de investigación; Ciudad, Ambiente y Tecnología (CAT), y Sistemas embebidos y visión artificial en ciencias, Arquitectónicas, Agropecuarias, Ambientales y Automática (SEVA4CA), por los conocimientos e información brindados para la elaboración del trabajo.

\section{Referencias bibliográficas}

Ahvenniemi, H., Huovila, A., Pinto-Seppä, I., \& Airaksinen, M. (2017). What are the differences between sustainable and smart cities? Cities, 60, 234-245.

Ameen, R. F. M., Mourshed, M., \& Li, H. (2015). A critical review of environmental assessment tools for sustainable urban design. Environmental Impact Assessment Review, 55, 110-125.

Dizdaroglu, D. (2015). Developing micro-level urban ecosystem indicators for sustainability assessment. Environmental Impact Assessment Review, 54, 119-124.

Dorantes Nova, J. A., Hernández Mosqueda, J. S., \& Tobón Tobón, S. (2016). Juicio de expertos para la validación de un instrumento de medición del síndrome de burnout en la docencia. Ra Ximhai, 327-346.

Elsa Guerrero, G., \& Cecilia Erbiti, C. (2004). Indicadores de sustentabilidad para la gestión de los residuos sólidos domiciliarios. Municipio de Tandil, Argentina. Revista de Geografía Norte Grande, (32), 71-86.

Escobar-Pérez, J., \& Cuervo-Martínez, Á. (2008). Validez De Contenido Y Juicio De Expertos: Una Aproximación a Su Utilización. Avances En Medición, 6, 27-36.

Ferronato, N., \& Torretta, V. (2019). Waste mismanagement in developing countries: A 
review of global issues. International Journal of Environmental Research and Public Health, 16(6).

Hák, T., Janoušková, S., \& Moldan, B. (2016). Sustainable Development Goals: A need for relevant indicators. Ecological Indicators, 60, 565-573.

Hassan, A. M., \& Lee, H. (2015). Toward the sustainable development of urban areas: An overview of global trends in trials and policies. Land Use Policy, 48, 199-212.

Hiremath, R. B., Balachandra, P., Kumar, B., Bansode, S. S., \& Murali, J. (2013). Indicator-based urban sustainability-A review. Energy for Sustainable Development, 17(6), 555-563.

Moschen, S. A., Macke, J., Bebber, S., \& Benetti Correa da Silva, M. (2019). Sustainable development of communities: ISO 37120 and UN goals. International Journal of Sustainability in Higher Education, 20(5), 887-900.

Quesada, M. F. (2018). Desarrollo de nuevos métodos de Evaluación Sustentable de la edificación a partir de la revisión del Estado del Arte. ACE: Architecture, City and Environment, 12(37), 51-70.

Quezada Ortega, V. P., Ávila Calle, M. B., \& Castillo Ortega, Y. (2021). Propuesta de lineamientos normativos para el uso del suelo rural en el caso del cantón Palora provincia de Morona Santiago. In Ciencia Digital (Vol. 5, Issue 1, pp. 114-132).

Sachs, J. D. (2012). From millennium development goals to sustainable development goals. The Lancet, 379(9832), 2206-2211.

United Nations, Department of Economic and Social Affairs, P. D. (2018). The World 's Cities in 2018. The World's Cities in 2018 - Data Booklet (ST/ESA/SER.A/417), 34.

Velásquez, J. (2013). Indicators of Sustainable Development for the Planification of Caroni Municipality. Universidad, Ciencia y Tecnología, (17), 19-27.

\section{Conflicto de intereses}

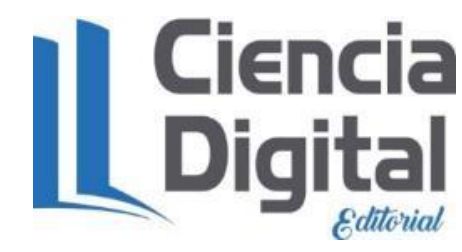

El presente artículo no presenta conflicto de intereses. 


\section{Conciencia}

El artículo que se publica es de exclusiva responsabilidad de los autores y no necesariamente reflejan el pensamiento de la Revista Conciencia Digital.

\section{LCiencia}

El artículo queda en propiedad de la revista y, por tanto, su publicación parcial y/o total en otro medio tiene que ser autorizado por el director de la Revista Conciencia Digital.
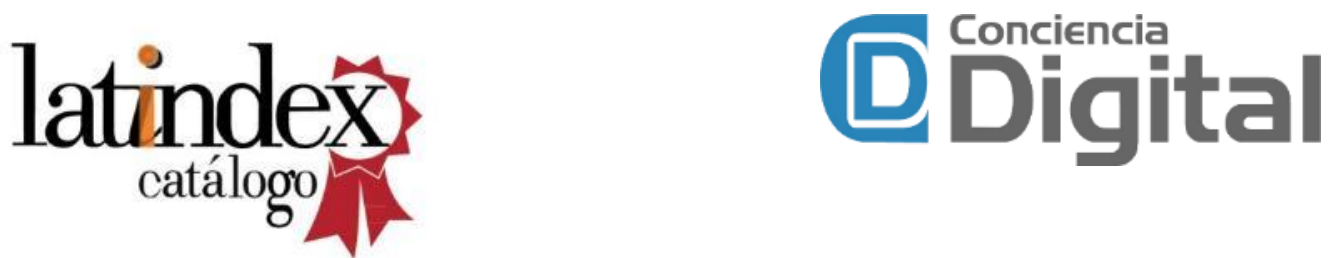

Indexaciones

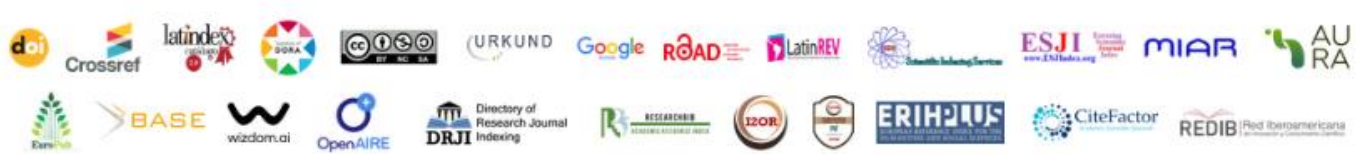

